IMAGE

\title{
Epilepsy? Video monitoring of long QT syndrome-related aborted sudden death
}

Tom Rossenbacker, MD, PhD, * Dieter Nuyens, MD, PhD, ${ }^{*}$ Wim Van Paesschen, MD, PhD, ${ }^{\dagger}$

Hein Heidbüchel, MD, PhD*

From the *Center for Hereditary Heart Diseases and Department of Cardiology, and Department of Neurology,

University of Leuven, Belgium.
(A)
1
2

EEG

Onset TdP

F3-G19

C3-G19

P3-G19

T2-G19

T1-G19

ECG

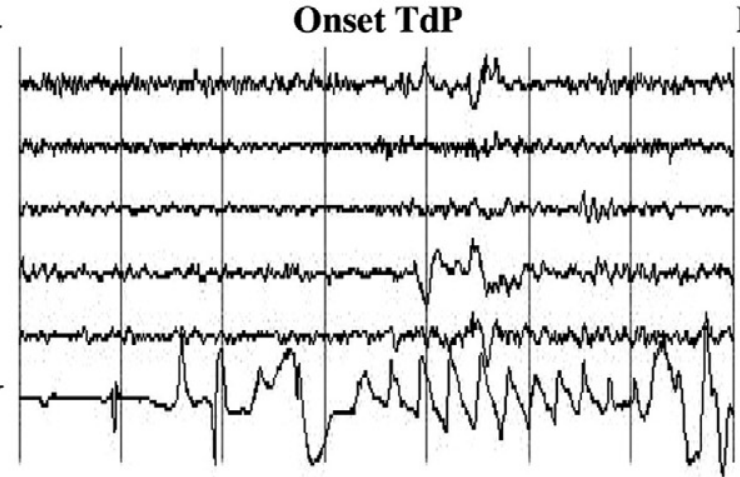

Electrocerebral silence developed 1'24" after onset TdP

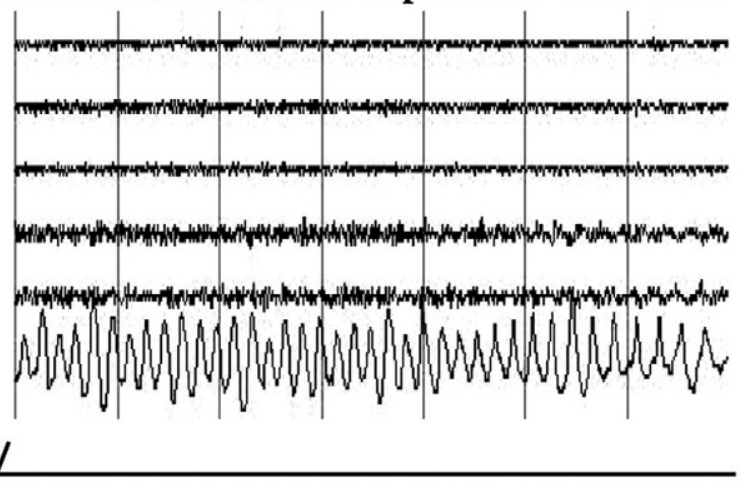

(B)

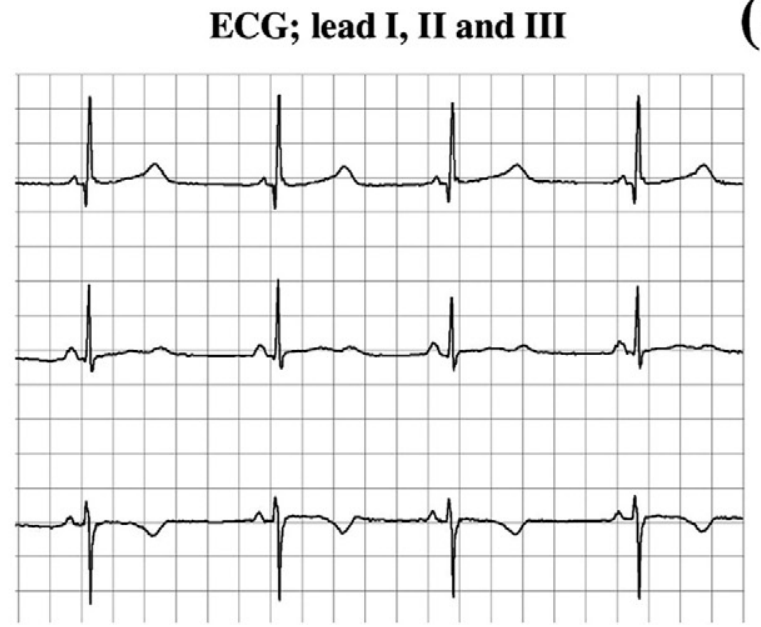

(C)

Holter recording
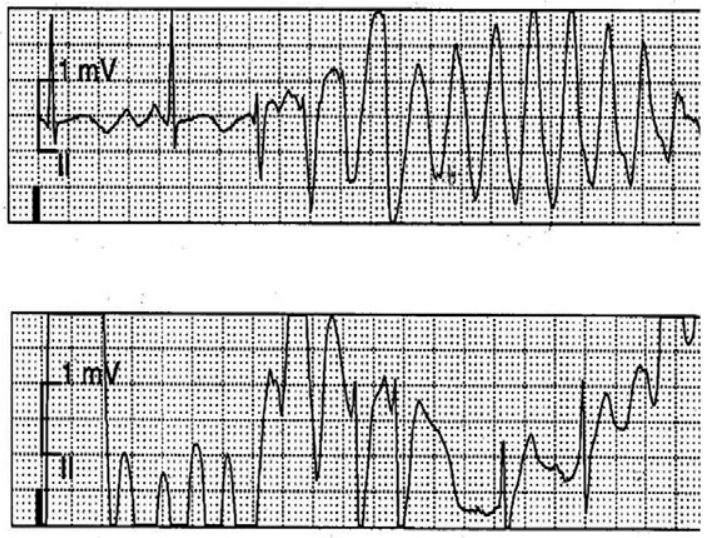

Figure 1

Address reprint requests and correspondence: Dr. Hein Heidbüchel, M.D., Ph.D., Cardiology-Electrophysiology, University Hospital Gasthuisberg, University of Leuven, Herestraat 49, B-3000 Leuven, Belgium. E-mail address: hein.heidbuchel@uz.kuleuven.ac.be.
A 15-year-old girl was referred to our center for an evaluation of medically refractory epilepsy. She had experienced generalized seizure attacks weekly since the age of 11 under treatment with anti-epileptics. We performed a 24-hour evaluation with combined electrocardiographic (ECG), electroencephalographic (EEG), and video recordings. Dur- 
ing the observation, the patient developed a convulsive seizure. Unexpectedly, neurological symptoms were provoked by a primary cardiac arrhythmia.

At onset of the symptoms, a polymorphic ventricular tachycardia was recorded, initiated by a postextrasystolic pause (Figure 1A, panel 1). The first EEG changes appeared 14 seconds after arrhythmia onset as polymorphic high-voltage delta activity (not shown), which deteriorated 1 minute, 24 seconds after clinical onset into a 14-second period of electrocerebral silence (Figure 1A, panel 2; muscular artifacts cause background noise). The patient spontaneously regained consciousness with normalization of the EEG and ECG.

The event prompted a cardiological evaluation. A baseline ECG showed normal sinus rhythm with a markedly prolonged QTc interval of $547 \mathrm{~ms}$ and low-amplitude widely split T waves (leads I, II, III, Figure 1B). During hospitalization, multiple runs of torsades de pointes (TdP) were observed, with a longest run of 1 minute, 54 seconds. One exemplary trace is depicted in Figure 1C. Although diagnosis of long QT syndrome (LQTS) was evident, it was not considered or detected during prior evaluations. The patient was started on a $\beta$-blocker and $\mathrm{K}^{+}$supplementation. Given the impressive symptomatic clinical presentation, we opted for the additional implantation of a prophylactic DDD implantable cardioverter-defibrillator. Under this therapy, she no longer developed TdP during an ensuing follow-up period of more than 2 years. No causative mutations were found in the KCNQ1, KCNH2, SCN5A, KCNE1, and KCNE2 genes.

LQTS-associated symptoms are often attributed incorrectly to a seizure disorder. Even in a tertiary neurological referral center, cardiac arrhythmias were not suspected as the cause of the seizures. Diagnosis of LQTS could have been made much earlier by including an ECG in the diagnostic approach of this patient presenting with syncopes. We hope this case may help educate those involved in syncope or "epilepsy" evaluation to identify life-threatening TdP early and promptly.

\title{
EP AROUND THE WORLD
}

\section{Electrophysiology around the world: Nepal}

\author{
Sujeeb Rajbhandari, MD, Man Bahadur, KC, MD, Ajay Naik, MD, DM, Roshan Raut, MD, \\ C. Thomas Peter, MD
}

From Shahid Gangalal National Heart Center, Bansbari, Kathmandu, Nepal.

Nepal is a small (area 143,000 $\mathrm{km}^{2}$ ) landlocked country situated in South Asia and is one of the poorest countries in the world. Its population is approximately 24.7 million, and the doctor/patient ratio is 1:10,000. People living in Nepal are largely dependent on farming, and the vast majority of the population lives below the poverty line. The population is multiracial and multilingual. The country currently is emerging from major political, economic, and social upheaval.

Shahid Gangalal National Heart Center (SGNHC) provides interventional cardiology and cardiac surgery facilities. For the entire country of Nepal, SGNHC is the only center with a cardiac electrophysiology (EP) laboratory. The first EP study with ablation was performed on October 13, 2003, in a temporary portable EP lab. The workshop lasted 5 days, and 12 patients benefited from the procedure. Since then, EP workshops have been run twice per year, and in 2005 the center purchased its own EP lab. To date, 191 procedures (EP study in 28 and ablations in 163) have been performed at SGNHC. Of the 163 ablations, 58 were performed for atrioventricular nodal reentrant tachycardia (AVNRT), 49 Wolff-Parkinson-White syndrome, 21 concealed accessory pathways, and 35 other.

Ethnic Nepalese patients are short statured, with mesomorphic and endomorphic physical features. As a result, the catheters used for the procedures require more acute curves. The ablation catheters used were predominantly short with small curves (e.g., Webster Green ablation catheter for left lateral accessory pathways and Webster Blue ablation catheter for slow pathway ablation of typical AVNRT). In many cases, nondeflectable diagnostic catheters were manually shaped to

Address reprint requests and correspondence: Dr. Sujeeb Rajbhandari, Shahid Gangalal National Heart Center, Bansbari, Kathmandu, Nepal. E-mail address: sujeebr@hotmail.com. increase the acuity of curves and shape them to the patient's cardiac anatomy.

The country's population is distributed in a few urban pockets (the only major city is the capital Kathmandu) and in large rural areas in the sub-Himalayan ranges and the southern plains. Some patients have suffered from episodes of supraventricular tachycardias that have sustained for a few days up to 1 week. Acute care and expertise in treating cardiac arrhythmias are available in only very limited locations. Some patients with ongoing tachycardia have traveled on foot and by animal conveyance for 3 to 5 days to reach a hospital for treatment. The fact that a procedure lasting 45 minutes (EP study and radiofrequency ablation) can permanently cure their condition is a watershed revelation in the lives of these individuals. Curative ablation has remarkably improved the quality of life of the majority of patients.

Of the 163 ablation cases, four patients had reappearance of delta waves on surface ECG with no documentation of palpitations. Two had recurrence of tachycardia. Twenty EP studies and 28 radiofrequency ablations were performed by the team comprising solely Nepalese cardiologists; two of these ablations were unsuccessful and were redone at a later date. No major complications were noted, except for one pseudoaneurysm of the right femoral artery.

Repeated admissions and hospitalizations for tachycardia poses a major financial burden on patients. Curative ablation has freed the majority of patients from these shackles; they are able to lead socially productive lives and are saved the financial expense of traveling abroad for ablation treatment. Although SGNHC is providing curative ablation therapy at nominal prices, even that cost is beyond the paying capacity of the majority of Nepalese people. 